\title{
Balancing Bias and Burden in Personal Network Studies
}

\author{
Marie Stadel $^{\mathrm{a}} \&$ Gert Stulp $^{\mathrm{a}}$ \\ ${ }^{a}$ Department of Sociology, University of Groningen, Grote Rozenstraat 31, 9712 TS Groningen, The \\ Netherlands
}

Acknowledgements: We would like to thank the Center for Information Technology of the University of Groningen for their support and for providing access to the Peregrine high performance computing cluster. Further, we are grateful for the valuable feedback of Casper Albers and Laura Bringmann. Last, we thank Marijtje van Duijn whose feedback on content and writing improved the manuscript considerably.

Funding: The LISS panel data were collected by CentERdata (Tilburg University, The Netherlands) through its MESS project funded by the Netherlands Organization for Scientific Research. This work was further supported by the Netherlands Organisation for Scientific Research (VENI Grant 451-15-034).

Declarations of interest: none

Corresponding author: m.stadel@rug.nl 


\begin{abstract}
Personal network data is increasingly used to answer research questions about the interplay between individuals (i.e., egos) and their social environment (i.e., alters). Researchers designing such data collections face a trade-off: When eliciting a high number of alters, study participation can be particularly burdensome as all data is obtained by surveying the ego. Eliciting a low number of alters, however, may incur bias in network characteristics. In the present study we use a sample of 701 Dutch women and their personal networks of 25 alters to investigate two strategies reducing respondent burden in personal network data collections: (1) eliciting fewer alters and (2) selecting a random subsample from the original set of elicited alters for full assessment. We present the amount of bias in structural and compositional network characteristics connected to applying these strategies as well as the potential study time gain for every possible network size ( 2 to 24 alters) as a proxy for respondent burden reduction. Our results can aid researchers designing a personal network study to balance respondent burden and bias in estimates for a range of compositional and structural network characteristics.
\end{abstract}

Keywords: personal networks; network delineation; respondent burden; parameter bias 


\section{Balancing Bias and Burden in Personal Network Studies}

The interplay between individuals and their social environment is becoming an increasingly important topic in behavioural science research (e.g. Kinderman et al., 2020). With rising interest in research questions about how characteristics of individuals' social environment influence their traits, cognitions and behaviour, or vice versa, social network data, particularly personal network data, is frequently collected (e.g. Aschbrenner et al., 2018; Rapp et al., 2019) and utilised in various contexts.

While whole social networks map out the ties in a well-defined closed social system (e.g. a school class), personal networks, also termed ego networks, contain information about one person's direct social connections (Perry et al., 2018). The central actor is termed the ego and the social connections are called alters. Rich data about the composition of the personal network (i.e. characteristics of alters and social relationships) as well as its structure (i.e. the ties between alters) is collected solely by surveying the ego (McCarty et al., 2019). A central concern in the literature regarding these data collections is the associated respondent burden (McCarty et al., 2007; Vehovar et al., 2008).

\section{Respondent Burden in Personal Network Data Collections}

When providing social network data, an ego first has to list names of alters (see Marsden, 2014). Subsequently, information about the elicited alters is assessed by asking the ego so-called name interpreter questions. These questions refer to alter or tie attributes (e.g., gender, type of relationship with the alter, or closeness to the alter) as well as relations between the different alters, termed alter-toalter ties (e.g., if alter $\mathrm{X}$ has contact with alter $\mathrm{Y}$ ).

Answering a large number of these questions usually takes long to complete and is repetitive. Therefore personal network data collections can be particularly burdensome for respondents (Vehovar et al., 2008). This can lead to compromised data quality (Hsieh, 2015; Manfreda et al., 2004; Matzat \& Snijders, 2010). The most prominent factors that may reduce respondent burden are (1) the personal network survey design, (2) the number and format of alter attribute questions and (3) the number of elicited alters. We will elaborate on these points below in more detail.

\section{Survey design}

To lower respondent burden it is important to design an efficient and easily understandable survey (Manfreda et al., 2004; Vehovar et al., 2008). One efficient design choice that reduces cognitive effort as well as study duration is to assess alter attributes question-wise rather than alterwise. In other words, asking respondents to rate all alters on one alter attribute rather than measuring all alter attributes for one alter at a time (Coromina \& Coenders, 2006; Lackaff, 2012; Vehovar et al., 2008). Due to the lowered burden, this approach also increases data quality (Vehovar et al., 2008). Another development in the field is the use of graphical features (Hogan et al., 2007; McCarty \& Govindaramanujam, 2005; McCarty, Molina, et al., 2007) and an interactive survey design (Eddens, 
Fagan \& Collins, 2017; Stark \& Krosnick, 2017; Tubaro et al., 2014, Stulp, 2020). Such modern designs make the data collection process more efficient and attractive, for example by asking respondents to draw the alter-to-alter ties into a network representation rather than assessing each tie with an individual question (McCarty \& Govindaramanujam, 2005). Respondents find these interactive survey designs with graphical interfaces easily understandable and more enjoyable than traditional assessment methods and they also seem to improve data quality by reducing mechanical answering behaviours (Stark \& Krosnick, 2017; Tubaro et al., 2014). However, these approaches do not seem to reduce the survey completion time.

\section{Alter Attribute Questions}

The number and format of alter attribute question evidently also impact respondent burden. For each additional alter attribute question, the question has to be answered as many times as there are number of alters. There also great differences in the time needed to response to the questions based on the format of questions. A recent study assessing personal networks with 25 alters by Stulp (2020) indicates that with an interactive and user-friendly survey design, assessing 16 alter attributes is on average considerably more time consuming than the alter elicitation and the alter-to-alter tie assessment combined (15.2 versus 7.1 minutes). Simple questions, for example 'indicate alters that are friends', which only required clicking on respective alters, could be completed reasonably fast ( $\sim 30$ seconds for 25 alters). Other questions, such as how close ego is to each of the alters, which required dragging each alter into a box, needed double the time to be completed ( 1-1.5 minutes for 25 alters). The most demanding alter questions, which required either the use of a radio button to indicate alter age or the evaluation of multiple checkboxes to indicate the type of relationship with the alter, took even longer ( 2-3 minutes for 25 alters). Thus, in order to minimise respondent burden, the number of alter attribute questions in general, and of more demanding ones in particular, should be kept to a minimum (McCarty, Killworth, et al., 2007).

\section{The number of alters}

Increasing the number of elicited alters increases respondent burden in three ways. First, additional alter names need to be generated. This may become particularly burdensome when asking for large(r) networks (e.g., > 5 alters), as the time it takes to list an individual alter is longer for later alters (Stulp, 2020). Second, for each alter, all alter attributes need to be assessed. Therefore, the total number of questions increases linearly with the number of. Third, the potential ties between the different alters need to be indicated. The number of these alter pair evaluations increases quadratically with the number of alters.

Previous studies (Golinelli et al., 2010; Stulp, 2020) indicate that the name generation phase can be completed for a large number of alters (e.g. 25) relatively fast (in $\sim 3.5$ minutes). The increasingly large number and repetitiveness of alter attribute and alter-to-alter tie questions can, however, impose an additional burden besides mere time investment and may also lead to motivational loss (Hsieh, 2015). This can significantly decrease data quality (Manfreda et al., 2004). The number of 
assessed alters are also associated with study drop-out rates (Vehovar et al., 2008). Therefore, different authors advise to limit the number of alters included in the network (e.g., Gerich \& Lehner, 2006; Manfreda et al., 2004).

\section{The Trade-Off Between Respondent Burden and Bias in Estimates}

While choosing a high number of alters to elicit may decrease data quality due to respondent burden, a number too low may lead to biased estimates. Small personal networks certainly do not contain so-called weak ties (Granovetter, 1973) and potentially even some stronger social contacts are not included (Marin \& Hampton, 2007). Therefore, the extent to which the assessed network represents the social environment of the ego can be distorted.

Hence, eliciting fewer alters can lead to biased estimates of both, structural (Costenbader \& Valente, 2003; McCarty, Killworth, et al., 2007) and compositional network characteristics (Marin \& Hampton, 2007). How strongly and in which direction measures are biased, however, varies considerably, not only between compositional and structural measures, but also between measures within one category (Marin \& Hampton, 2007; McCarty et al., 2007). Given that no reliable inferences can be drawn when using biased measures of network structure and composition, a balance needs to be found between the respondent burden, i.e., the study duration mainly influenced by the number of alters in the study, and the bias incurred in estimates of network characteristics.

\section{Overcoming the Trade-Off}

A proposed strategy to reduce respondent burden without compromising estimates is to initially elicit a large number of alters in the name generation phase, but only assess alter attributes and alter-to-alter ties for a random subset of those alters (Manfreda et al., 2004; Marin \& Hampton, 2007). Marin and Hampton (2007) termed this strategy multiple generator random interpreter (MGRI) and found first evidence for its effectiveness in obtaining precise estimates of network measures while assessing fewer alters (6 instead of around 13 alters).

McCarty and colleagues (2007) tested MGRI as well as three other ways of reducing respondent burden in personal network studies: (1) assessing fewer alters, (2) randomly selecting a subset of specific alter-to-alter ties for evaluation and (3) predicting alter-to-alter ties based on transitivity. Eliciting fewer alters (25 instead of 45) appeared to be a feasible strategy since 25 alters still sufficiently captured the structural network characteristics of their data. An even more promising strategy that reduced respondent burden while minimising estimate bias, was eliciting a larger number of alters but then randomly selecting a subset of alters for whom attribute questions and ties were to be evaluated. This strategy provided reasonable estimates of almost all measures with 45 generated names of alters but as few as 10 fully assessed alters. The remaining two strategies that McCarty and colleagues tested biased estimates severely and are therefore not recommended. 
Golinelli and colleagues (2010) further investigated MGRI using a synthetic network with 20 alters to specifically quantify the bias of simulated structural measures (i.e. density, percentage of isolates, maximum degree and degree centralization) in terms of RMSE when assessing different network sizes (5 to 19 alters). From their simulation they concluded that the estimation error increases slower after at least half of all alters (i.e. 10-12) are sampled.

In addition, Golinelli and colleagues (2010) used partial data from a personal network survey of homeless women (see Ryan et al., 2009) to quantify the estimation error as well as the respondent burden in terms of study duration in 28 different networks. To obtain these 28 networks 20 alters were elicited, but 14 alter attribute questions and the alter-to-alter ties were only assessed for a random sample of 12 alters. Their results indicated that including alter-to-alter tie information of 12 to 19 alters led to up to $10 \%$ error of structural network characteristics, while the error sharply increased when including less than 12 alters.

\section{This Study}

The current study adds to previous work by (1) using a representative sample, (2) investigating the burden (i.e., study time) connected to assessing alter attributes, the part of personal network data collection that is often the lengthiest, and (3) quantifying the amount of bias in compositional measures, which are often key variables used in personal network studies.

Specifically, we use representative data from a sample of Dutch women of ages 18 to 40 and their personal networks (of 25 alters) to show the trade-off between the bias in structural and compositional network estimates and the number of alters that are assessed (Buijs \& Stulp, 2019; Stulp, 2020). The two most successful strategies to reduce respondent burden identified by McCarty and colleagues (2007) are applied: (1) dropping alters completely and (2) generating a larger number of alters (i.e. 25) but only assessing a random subset of them.

Additionally, estimated time savings for slow, fast and average respondents connected to applying these two methods are presented. This information enables researchers to make informed decisions about minimizing the respondent burden of their personal network data collection while still obtaining sufficiently reliable estimates of structural and compositional network characteristics. 


\section{Methods}

\section{Data}

The current study uses data of a representative sample of Dutch women $(N=758$; age range 18-40) and their personal networks with 25 alters obtained via the LISS (Longitudinal Internet Studies for the Social sciences) panel (Scherpenzeel, 2011; Stulp, 2020). The personal network data was collected with a modified version of GENSI (Stark \& Krosnick, 2017), a recently developed tool for assessing personal network data using interactive graphical elements. In addition to the elicitation of 25 alters, respondents were asked to answer 16 attribute questions about these alters, as well as all 300 alter-to-alter ties. For the present study only 9 attribute questions were considered, as these questions were deemed generalisable across different study contexts (see Table 1). A full description of the data collection procedure, the representative sampling of the LISS panel and the complete survey can be found in Stulp (2020). Data will become available on https://www.lissdata.nl/.

\section{Personal Network Data}

For the present study participants were only included if they (1) filled in exactly 25 alters, (2) had no more than 10 missing responses on alter attribute questions, (3) completed the survey on a computer as instructed rather than on a phone or tablet (GENSI was optimised for use on computer), (4) listed at least one alter-to-alter tie. These inclusion criteria resulted in a final dataset containing 701 personal networks.

\section{Study Duration Data}

Respondents who took breaks while filling out the survey can still provide valid network data, they, however, should not be considered when determining a representative study duration. Therefore, only respondents who took less than 10 minutes per question were included for the duration calculations $(N=649$; see also Stulp, 2020). Based on this sample, we determined study durations of all parts for the average respondent, the $10^{\text {th }}$ percentile (i.e., the $10 \%$ fastest respondents) and the $90^{\text {th }}$ percentile (i.e., the $10 \%$ slowest respondents). The total study duration is composed of the duration that it takes respondents to (1) generate all alter names, (2) answer the nine alter attribute questions, and (3) evaluate all alter-to-alter ties.

For the name generation, precise data indicating how long each ego took to name each alter was available. We averaged the duration for naming each separate alter across the 649 egos and added these durations to determine how long it took all respondents on average to name 1 to 25 alters. For example, to determine the duration for naming 2 alters the average time it took all respondents to name the first alter was added to the average time it took them to name the second one. The same procedure was followed for the $10 \%$ fastest and slowest respondents to give some sense of how the different strategies impact respondent burden for different groups of respondents.

For all name interpreter questions, only the total time that it took each ego to answer the respective question for all 25 alters was available (e.g., the time it took an ego to indicate the gender of 
all alters). In case of name interpreter questions for which respondents had to necessarily evaluate each alter separately (i.e., gender, age, relationship type, closeness, education, contact frequency, alter children, alter-to-alter tie evaluation) we determined the time for assessing one alter by averaging the duration of the given question across the 649 egos and divided it by 25 . Therefore, we assume that answering the question took equally long for each alter. This time is then multiplied by the number of alters to determine the duration of this question for each sample size. For example, on average it took respondents about 28 seconds to indicate the gender of all 25 alters. From this we calculated that indicating the gender of one alter took about 1.12 seconds, for two alters 2.24 seconds and so on.

One name interpreter question (i.e., indicate friends) did not necessarily require separate actions for each alter. Therefore, assuming that it would take equally long to answer the question with respect to each alter is less realistic. For this question, the mean duration across egos for assessing 25 alters (i.e. 34 seconds) is taken regardless of the number of alters. This most likely results in an overestimation of the study duration when selecting fewer alters. Again, the same procedure was repeated for the $10 \%$ fastest and slowest respondents.

\section{Network Measures}

The structural measures that we investigated are the most common measures used in the literature: network density, the proportion of isolates, maximum degree centrality, mean and maximum closeness centrality, mean and maximum betweenness centrality as well as degree, closeness and betweenness centralisation. Measures that are sensitive to the number of alters in the network were normalised according to Wassermann and colleagues (1994).

We investigated the compositional network measures summarizing the assessed alter attributes. The resulting measures fall into two categories: demographic composition and role relationships/tie characteristics. We used averages and standard deviations to summarise (approximately) continuous alter attributes and proportions for categorical alter attributes. In order to be able to compare characteristics measured on a different scale to each other, characteristics were normalised to a scale from 0 to 1 . 
Table 1

Alter Attribute Questions

\begin{tabular}{|c|c|c|}
\hline Item & Answer options & Response format \\
\hline gender & male or female & click on alter \\
\hline age & $18,19, \ldots, 49,50,50+$ & radio button \\
\hline type of relationship ${ }^{I}$ & $\begin{array}{l}\text { partner, parent, siblings, other relative, relative of } \\
\text { partner, acquaintance/friend of partner, from primary } \\
\text { school, from college/university, from work, from a social } \\
\text { activity, through a mutual acquaintance/friend, from the } \\
\text { neighbourhood, and other }\end{array}$ & $\begin{array}{l}\text { checkboxes, } \\
\text { multiple answers } \\
\text { possible }\end{array}$ \\
\hline closeness & $\begin{array}{l}\text { very close }(=1), \text { close, somewhat close, not close, really } \\
\text { not close }(=5)\end{array}$ & $\begin{array}{l}\text { drag alters into } \\
\text { boxes }\end{array}$ \\
\hline education & $\begin{array}{l}\text { Primary school or hasn't finished primary school }(=1) \text {, } \\
\text { High-school diploma (or a similar diploma), Secondary } \\
\text { vocational education (or a similar diploma), Higher } \\
\text { vocational education (or a similar diploma), University } \\
\text { degree or higher (or a similar diploma) }(=5)\end{array}$ & $\begin{array}{l}\text { drag alters into } \\
\text { boxes }\end{array}$ \\
\hline $\begin{array}{l}\text { frequency of face-to-face } \\
\text { contact }\end{array}$ & $\begin{array}{l}\text { Daily }(=1) \text {, A couple of times per week, A couple of } \\
\text { times per month, About once a month, A couple of times } \\
\text { per month or less }(=5)\end{array}$ & $\begin{array}{l}\text { drag alters into } \\
\text { boxes }\end{array}$ \\
\hline $\begin{array}{l}\text { frequency of other forms of } \\
\text { contact }\end{array}$ & $\begin{array}{l}\text { Daily }(=1) \text {, A couple of times per week, A couple of } \\
\text { times per month, About once a month, A couple of times } \\
\text { per month or less }(=5)\end{array}$ & $\begin{array}{l}\text { drag alters into } \\
\text { boxes }\end{array}$ \\
\hline $\begin{array}{l}\text { which of these people do } \\
\text { you consider a friend? }\end{array}$ & friend or not a friend & click on alter \\
\hline does alter $x$ have children? & yes or no & click on alter \\
\hline
\end{tabular}

${ }^{1}$ Only the proportion of kin and of friends was considered. 


\section{Emulating Burden Reduction Strategies}

All data preparations, emulations, analyses and visualizations were done using $\mathrm{R}$ ( $\mathrm{R}$ Core Team, 2018). We used the following packages: broom (Robinson, 2014), ggraph (Thomas Lin Pedersen, 2017), ggplot2 (Wickham, 2016), Hmisc (Harrell Jr, 2019), igraph (Csardi, 2013), knitr (Xie, 2014), lme4 (Bates et al., 2015), patchwork (Pedersen, 2019), tidygraph (Pedersen, 2018), tidyverse (Wickham, 2017) and skimr (Waring et al., 2020). All analysis scripts be found on DataVerseNL (https://doi.org/10.34894/JNVWNH).

We calculated all network measures using the full 25 alter networks. These estimates are considered the 'true' compositional and structural measures for the purpose of this study. We calculated descriptive statistics of these measures across the 701 personal networks.

\section{Dropping Alters Completely}

To investigate the strategy of dropping alters completely, all compositional and structural network characteristics were calculated for each sample size ( 2 to 24 alters) for each ego by selecting the respective alters from the top of each generated alter list. For example, to emulate the assessment of only 10 alters, we only considered the data connected to the first 10 alters that an ego generated. Based on this sample, all structural and compositional measures were calculated. This procedure was completed for each ego for each sample size (2 to $\left.24^{\mathrm{i}}\right)$.

\section{Determining Bias}

We then determined the incurred bias by subtracting the true value of the network measure from the sample estimate. Thus, a positive bias value indicates overestimating the measure in the sample while negative errors signify underestimating. Besides these raw errors (i.e. bias), we also determined absolute error values.

\section{Determining Burden}

We operationalised respondent burden reduction as the total study time gain connected to eliciting fewer alters. We calculated the study time gain for the average respondent of by subtracting the average study duration of the reduced network assessment (e.g., when dropping the last 15 alters) from the total average study duration (i.e. when generating 25 alters and also assessing all questions for all 25 alters). When for example dropping the last 15 alters (i.e., only eliciting the first 10 alters), the average duration of generating 10 alters was added to the average durations of answering each name interpreter question for 10 alters. We repeated this procedure for the $10 \%$ fastest and slowest respondents.

\section{Randomly Sampling Alters for Full Assessment}

We, again, considered all network sizes, ranging from 2 to 24 alters, with the number of alters denoted by $n$. Similar to Golinelli and colleagues (2010), we drew 1000 samples ${ }^{\mathrm{ii}}$ of $\mathrm{n}$ alters from each of the full personal networks. For network sizes of 2, 23 and 24 alters, we differed from this number of samples, since there are less than 1000 combinations of alters possible. Thus, for these three network 
sizes, we considered every possible combination of alters, which leads to 300,300 and 25 emulations for these network sizes, respectively. For each emulated sample, all network characteristics were estimated. Due to high computation time, emulations and error computations were run on the Peregrine HP Cluster (Peregrine Documentation, 2020).

Determining Bias

In accordance with the work of Golinelli and colleagues (2010) we calculated bias by taking the difference between the true network measure and the average of the emulated network measures of the same sample size. Following methods of these authors further, we calculated the root mean squared error (RMSE) by summarizing the squared errors across the different emulations per sample size.

\section{Determining Burden}

We operationalised respondent burden reduction as the total study time gain connected to assessing fewer alters fully. Again, we calculated this study time gain by subtracting the average study duration of the reduced network assessment (e.g., when randomly sampling 10 alters) from the total average study duration. For example, when randomly sampling 10 alters from a generated list of 25 alters, the average duration of eliciting 25 alters was added to the average duration of answering each name interpreter question for 10 alters. Also for this burden reduction strategy we calculated time gains not just for the average respondent, but also the $10 \%$ fastest and slowest respondents.

\section{Shiny app}

Because of the multitude of emulation results, using two different strategies of reducing burden, varying network size, and applying those to 23 different outcomes, presentation and elaborate discussion of each of these outcomes would be beyond the scope of this article. Instead, we focus on patterns that hold across outcomes, and provide a more detailed walk-through on density (which is a frequent measure of interest). However, different researchers may be interested in different outcomes, which is why we also provide an accompanying Shiny app (https://socialsciencemethods.shinyapps.io/BalancingBiasAndBurden) that allows readers to access the full results relevant to their research. 


\section{Results}

\section{Descriptives of the Personal Network Characteristics}

Descriptive statistics of all 'true' network measures across the 701 personal networks containing all 25 alters can be found in Table 2. As can be seen, the sample of personal networks provides a range of the investigated structural and compositional network characteristics. Further interpretation of the descriptive statistics is not the aim of the current paper.

\section{Table 2}

Descriptive Statistics of Network Measures Across All Personal Networks

\begin{tabular}{|c|c|c|c|c|c|}
\hline & Minimum & Mean & Median & Maximum & $\begin{array}{l}\text { Standard } \\
\text { Deviation }\end{array}$ \\
\hline Density & 0.02 & 0.24 & 0.23 & 0.81 & 0.11 \\
\hline Proportion of Isolates & 0 & 0.07 & 0.04 & 0.72 & 0.10 \\
\hline Maximum Degree & 0.02 & 0.33 & 0.30 & 0.82 & 0.16 \\
\hline Degree Centralisation & 0.12 & 0.57 & 0.54 & 1 & 0.21 \\
\hline Betweenness Centralisation & 0 & 0.26 & 0.21 & 0.90 & 0.20 \\
\hline Mean Betweenness Centrality & 0 & 0.02 & 0.02 & 0.16 & 0.02 \\
\hline Maximum Betweenness & 0 & 0.27 & 0.23 & 0.90 & 0.20 \\
\hline \multicolumn{6}{|l|}{ Centrality } \\
\hline Closeness Centralisation & 0 & 0.16 & 0.07 & 0.94 & 0.21 \\
\hline Mean Closeness Centrality & 0.04 & 0.20 & 0.12 & 0.86 & 0.18 \\
\hline Maximum Closeness Centrality & 0.05 & 0.28 & 0.15 & 1 & 0.27 \\
\hline Average Alter Age & 18.57 & 30.84 & 31.32 & 45.14 & 5.56 \\
\hline SD Alter Age & 0.53 & 6.06 & 5.99 & 14.20 & 2.69 \\
\hline Proportion of Female Alters & 0 & 0.64 & 0.64 & 1 & 0.13 \\
\hline Average Education & 1.40 & 3.43 & 3.40 & 5 & 0.62 \\
\hline SD Education & 0 & 0.89 & 0.88 & 1.70 & 0.26 \\
\hline Proportion of Friends & 0 & 0.42 & 0.40 & 1 & 0.21 \\
\hline Proportion of Kin & 0 & 0.38 & 0.36 & 0.92 & 0.18 \\
\hline Average Closeness & 1 & 2.52 & 2.48 & 4.48 & 0.47 \\
\hline SD Closeness & 0 & 1.06 & 1.05 & 1.86 & 0.24 \\
\hline Average In-Person Contact & 1.20 & 3.14 & 3.12 & 4.72 & 0.60 \\
\hline SD In-Person Contact & 0 & 1.16 & 1.17 & 1.88 & 0.25 \\
\hline Average Not In-Person Contact & 1 & 3.16 & 3.20 & 4.68 & 0.58 \\
\hline SD Not In-Person Contact & 0 & 1.19 & 1.21 & 1.95 & 0.26 \\
\hline
\end{tabular}

Note. $N=701$. Compositional network estimates are not yet normalised, but on their original scales as described in Table 1. 


\section{Emulations of Burden Reduction Strategies}

In the following, we will give a general overview of patterns in the results for all network measures. Interpreting all results for every measure in detail would move beyond the space restrictions for this paper. We will only explain the results for density extensively, since this is a common measure used in the literature. This explanation serves as a guideline to interpret the error plots of all investigated measures that can be found in our shiny app (https://socialsciencemethods.shinyapps.io/BalancingBiasAndBurden/).

Table 3 gives an overview of bias for each network characteristic across the 701 personal networks when applying each burden reduction method including 5, 10, 15 and 20 alters. Several observations can be made from this table. First, as expected, lowering the number of alters increases median bias for both methods. While median bias is comparatively low when considering 20 alters, including only 5 alters in a personal network data collection leads to less precise estimates of network measures regardless of the applied strategy.

Second, when dropping alters, median bias rises more steeply than when randomly sampling alters. Dropping the last 5 alters and randomly sampling 20 from 25 elicited alters leads to a similar average degree of error for most measures. Dropping the last 20 alters, however, can lead to considerably larger average errors than randomly sampling 5 from 25 elicited alters.

Third, median bias when randomly sampling alters shows naturally less variability (i.e., a lower median absolute deviation) between the 701 different personal networks than median bias when dropping alters completely. It is important to note, that this is also due to the fact that bias when randomly sampling indicates the centre of the distribution of 1000 values and does not reflect a single raw error calculation (as is the case when dropping alters).

Last, median bias differs largely between different network measures, with structural measures being more difficult to estimate than compositional ones. Maximum degree and degree centralisation are particularly problematic. In order to accurately estimate these two characteristics, randomly sampling alters is clearly the better choice than dropping alters. For other structural measures (i.e., the proportion of isolates and maximum betweenness centrality) dropping alters delivers lower median bias when randomly sampling alters at all network sizes. For all compositional measures, randomly sampling 20 alters provides less biased estimates than dropping the last 5 alters completely. Particularly for the proportion of kin in the network, average closeness to alters and average contact frequency with alters, dropping alters completely biases estimates considerably more than random sampling. 


\section{Table 3}

Median (Median Absolute Deviation) Bias in Network Characteristics Across All Personal Networks

\begin{tabular}{|c|c|c|c|c|c|c|c|c|c|}
\hline & \multirow[b]{2}{*}{$\begin{array}{c}\text { Median True } \\
\text { Value }\end{array}$} & \multicolumn{2}{|c|}{5 alters } & \multicolumn{2}{|c|}{10 alters } & \multicolumn{2}{|c|}{15 alters } & \multicolumn{2}{|c|}{20 alters } \\
\hline & & $\begin{array}{l}\text { Dropping } \\
\text { alters } \\
\text { (one } \\
\text { emulation) }\end{array}$ & $\begin{array}{c}\text { Random } \\
\text { sampling } \\
\text { (1000 } \\
\text { emulations })\end{array}$ & $\begin{array}{l}\text { Dropping } \\
\text { alters } \\
\text { (one } \\
\text { emulation) }\end{array}$ & $\begin{array}{c}\text { Random } \\
\text { sampling } \\
(1000 \\
\text { emulations })\end{array}$ & $\begin{array}{l}\text { Dropping } \\
\text { alters } \\
\text { (one } \\
\text { emulation) }\end{array}$ & $\begin{array}{l}\text { Random } \\
\text { sampling } \\
(1000 \\
\text { emulations })\end{array}$ & $\begin{array}{l}\text { Dropping } \\
\text { alters } \\
\text { (one } \\
\text { emulation) }\end{array}$ & $\begin{array}{c}\text { Random } \\
\text { sampling } \\
\text { (1000 } \\
\text { emulations) }\end{array}$ \\
\hline Density & 0.23 & $0.44(0.31)$ & $0(0)$ & $0.18(0.15)$ & $0(0)$ & $0.08(0.09)$ & $0(0)$ & $0.03(0)$ & $0(0)$ \\
\hline Proportion of Isolates & 0.04 & $0(0.06)$ & $0.33(0.12)$ & $0(0.06)$ & $0.13(0.07)$ & $0(0.04)$ & $0.05(0.04)$ & $0(0.01)$ & $0.02(0.02)$ \\
\hline Maximum Degree & 0.30 & $0.54(0.22)$ & $-0.14(0.13)$ & $0.39(0.20)$ & $-0.08(0.09)$ & $0.30(0.17)$ & $-0.04(0.05)$ & $0.26(0.12)$ & $-0.02(0.03)$ \\
\hline Degree Centralisation & 0.54 & $-0.40(0.27)$ & $-0.14(0.13)$ & $-0.27(0.20)$ & $-0.07(0.08)$ & $-0.25(0.16)$ & $-0.04(0.05)$ & $-0.24(0.13)$ & $-0.02(0.03)$ \\
\hline Betweenness Centralisation & 0.21 & $0.45(0.34)$ & $-0.12(0.16)$ & $0.15(0.16)$ & $-0.08(0.12)$ & $0.06(0.07)$ & $-0.04(0.09)$ & $0.02(0.02)$ & $-0.01(0.04)$ \\
\hline Mean Betweenness Centrality & 0.02 & $0.45(0.44)$ & $0(0.01)$ & $0.17(0.21)$ & $0(0.01)$ & $0.07(0.10)$ & $0(0.01)$ & $0.02(0.03)$ & $0(0)$ \\
\hline Maximum Betweenness Centrality & 0.23 & $0.08(0.23)$ & $-0.13(0.18)$ & $0.07(0.14)$ & $-0.08(0.13)$ & $0.03(0.06)$ & $-0.04(0.09)$ & $0.01(0.02)$ & $-0.01(0.05)$ \\
\hline Closeness Centralisation & 0.07 & $0(0.04)$ & $0.06(0.05)$ & $0.01(0.03)$ & $0.02(0.03)$ & $0.01(0.01)$ & $0.01(0.02)$ & $0(0.01)$ & $0(0.01)$ \\
\hline Mean Closeness Centrality & 0.12 & $-0.11(0.21)$ & $0.17(0.06)$ & $-0.03(0.15)$ & $0.06(0.04)$ & $0(0.10)$ & $0.03(0.02)$ & $0(0.06)$ & $0.01(0.01)$ \\
\hline Maximum Closeness Centrality & 0.15 & $-0.10(0.20)$ & $0.18(0.08)$ & $-0.03(0.14)$ & $0.07(0.05)$ & $0(0.10)$ & $0.03(0.03)$ & $0(0.05)$ & $0.01(0.01)$ \\
\hline Average Alter Age & 31.32 & $-0.01(0.09)$ & $0(0)$ & $-0.01(0.06)$ & $0(0)$ & $0(0.04)$ & $0(0)$ & $0(0.03)$ & $0(0)$ \\
\hline SD Alter Age & 5.99 & $-0.04(0.08)$ & $-0.01(0.01)$ & $-0.02(0.06)$ & $-0.01(0)$ & $-0.01(0.04)$ & $0(0)$ & $0(0.02)$ & $0(0)$ \\
\hline Proportion of Female Alters & 0.64 & $-0.08(0.18)$ & $0(0.01)$ & $-0.02(0.12)$ & $0(0)$ & $0(0.10)$ & $0(0)$ & $0(0.06)$ & $0(0)$ \\
\hline Average Education & 3.40 & $-0.01(0.12)$ & $0(0)$ & $-0.01(0.07)$ & $0(0)$ & $0(0.04)$ & $0(0)$ & $0(0.03)$ & $0(0)$ \\
\hline SD Education & 0.88 & $-0.01(0.08)$ & $-0.01(0.01)$ & $0(0.05)$ & $0(0)$ & $0(0.03)$ & $0(0)$ & $0(0.01)$ & $0(0)$ \\
\hline Proportion of Friends & 0.40 & $0.04(0.36)$ & $0(0.01)$ & $0.08(0.21)$ & $0(0)$ & $0.07(0.10)$ & $0(0)$ & $0.04(0.06)$ & $0(0)$ \\
\hline Proportion of Kin & 0.36 & $0.36(0.30)$ & $0(0.01)$ & $0.18(0.21)$ & $0(0)$ & $0.09(0.12)$ & $0(0)$ & $0.04(0.06)$ & $0(0)$ \\
\hline Average Closeness & 2.48 & $-0.28(0.12)$ & $0(0)$ & $-0.18(0.09)$ & $0(0)$ & $-0.10(0.06)$ & $0(0)$ & $-0.04(0.03)$ & $0(0)$ \\
\hline SD Closeness & 1.05 & $-0.13(0.11)$ & $-0.01(0)$ & $-0.06(0.08)$ & $0(0)$ & $-0.03(0.05)$ & $0(0)$ & $-0.01(0.02)$ & $0(0)$ \\
\hline Average In-Person Contact & 3.12 & $-0.24(0.15)$ & $0(0)$ & $-0.12(0.11)$ & $0(0)$ & $-0.07(0.07)$ & $0(0)$ & $-0.03(0.04)$ & $0(0)$ \\
\hline SD In-Person Contact & 1.17 & $-0.06(0.12)$ & $-0.01(0)$ & $-0.02(0.07)$ & $0(0)$ & $-0.01(0.05)$ & $0(0)$ & $0(0.02)$ & $0(0)$ \\
\hline Average Not In-Person Contact & 3.20 & $-0.28(0.16)$ & $0(0)$ & $-0.17(0.10)$ & $0(0)$ & $-0.10(0.06)$ & $0(0)$ & $-0.04(0.04)$ & $0(0)$ \\
\hline SD Not In-Person Contact & 1.21 & $-0.08(0.13)$ & $-0.01(0)$ & $-0.03(0.08)$ & $0(0)$ & $-0.01(0.05)$ & $0(0)$ & $0(0.02)$ & $0(0)$ \\
\hline
\end{tabular}

Note. $N=701$; Bias when dropping alters reflects the difference between the true network measure and one emulated value, bias when randomly sampling alters reflects the

difference between the true network measure and the average of 1000 emulated values; Median values of the true network measure are displayed in Table 2 . The scales of compositional characteristics can be found in Table 1. 


\section{Detailed example 'density' and study time savings}

Figure 1a and $1 \mathrm{~b}$ show the absolute error and bias incurred in network density when dropping alters completely. Figures 1c and 1d display the RMSE and bias incurred in network density when randomly sampling alters for full assessment. The secondary $\mathrm{x}$-axis of the presented plots also displays the potential time savings for average respondents connected to the two burden reduction methods. Readers can access all plots for their measure of interest in our shiny app and use this example to guide their interpretations. The app also provides the option to change the secondary $\mathrm{x}$-axis to view time savings for the $10 \%$ fastest and slowest respondents from our dataset.

In Figure 1a it can be seen that when including fewer alters in the network the median error rises more steeply, with 8 alters already delivering error rates of 0.25 , which is a quarter of the total range of the measure. Figure $1 \mathrm{~b}$ shows that density is overestimated (i.e., biased in the positive direction) for most of the 701 personal networks when dropping alters completely. Note for both of these plots, that the variability between the different personal networks is also increasing when fewer alters are included. This means that the fewer alters are sampled, the more difficult it is to give general recommendations since bias varies more between different networks.

The variability between networks is smaller when randomly sampling alters (Figures 1c and 1d) due to the inherent methodological difference in testing the two burden reduction strategies (i.e., obtaining one versus multiple error values; see methods). Figure 1c further shows that the RMSE connected to randomly sampling alters rises slowly with less than 0.1 median RMSE until about 9 alters, thus allowing to collect fewer alters. Figure $1 \mathrm{~d}$ additionally shows, that on average randomly sampling alters, does not bias density in a particular direction.

The secondary $\mathrm{x}$-axis of each plot displays the potential time saving when including 2 to 24 instead of 25 alters in the data collection. These time savings differ between the two methods since when dropping an alter completely, the name does not need to be generated and subsequently no further questions about this alter are asked. When randomly sampling alters, only the assessments of alter attribute questions and alter-to-alter ties are omitted, but still all alter names need to be generated. Therefore, time gains are larger when dropping alters completely. When for example dropping the last 5 alters, 233 seconds (i.e., nearly 4 minutes) of study time can be saved. When eliciting 25 alters but only fully assessing a random sample of 20 alters, only 177 seconds (i.e., nearly 3 minutes) can be saved. With a network size of 15 alters, dropping alters saves approximately $71 / 2$ minutes while randomly sampling alters saves 6 minutes (i.e., a difference of $1 \frac{1 / 2}{2}$ minutes). With 10 alters, the time difference between the two methods rises to about 2 minutes. The additionally invested time, however, also on average leads to less biased estimates of the network density.

These estimates on saving time are based on the average respondent. Researchers might consider samples that are faster (e.g., young, internet-savvy respondents) or slower (e.g., older respondents or patient population), which is why we also present estimates for the $10 \%$ fastest and slowest respondents of our sample. When completely dropping the last 5 alters, 112 seconds (i.e., 
about 2 minutes) of study time can be saved for the fastest respondent, whereas the slowest respondent experience a considerably higher time reduction of 384 seconds (i.e., almost $6 \frac{1}{2} 2$ minutes). When eliciting 25 alters but only fully assessing a random sample of 20 alters the differences between the groups are less pronounced: 104 seconds (i.e., nearly 2 minutes) can be saved for the fast group, and 264 seconds (i.e., $4 \frac{1}{2} 2$ minutes) for the slow group. With a network size of 15 alters, dropping alters saves approximately 4 minutes (fast) or 12 minutes (slow) while randomly sampling alters saves $3 \frac{1}{2}$ (fast) or 9 minutes (slow). Considering 10 alters, the slowest group can even save almost 18 minutes of study time when dropping alters completely and 13 minutes when randomly sampling alters. For the fast group the study time gains are at $51 / 2$ and 5 minutes, respectively. These results indicate that alter name generation required a substantial amount of time, especially for slow respondents. 


\section{Figure 1}

Error (left panels) and Bias (right panels) Incurred in Density When Dropping Alters Completely (Top) And Randomly Sampling Alters for Full Assessment (Bottom)
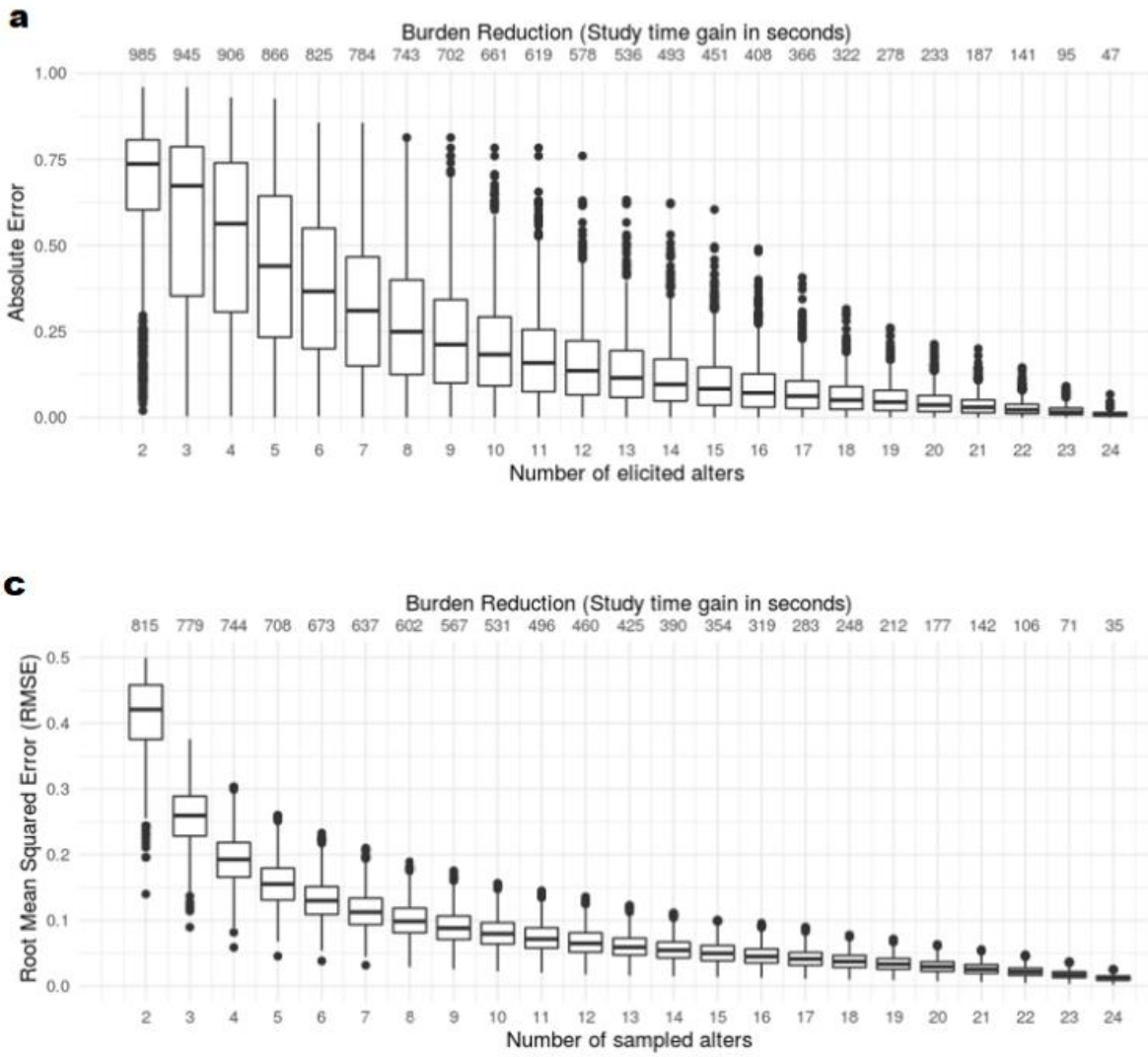

b

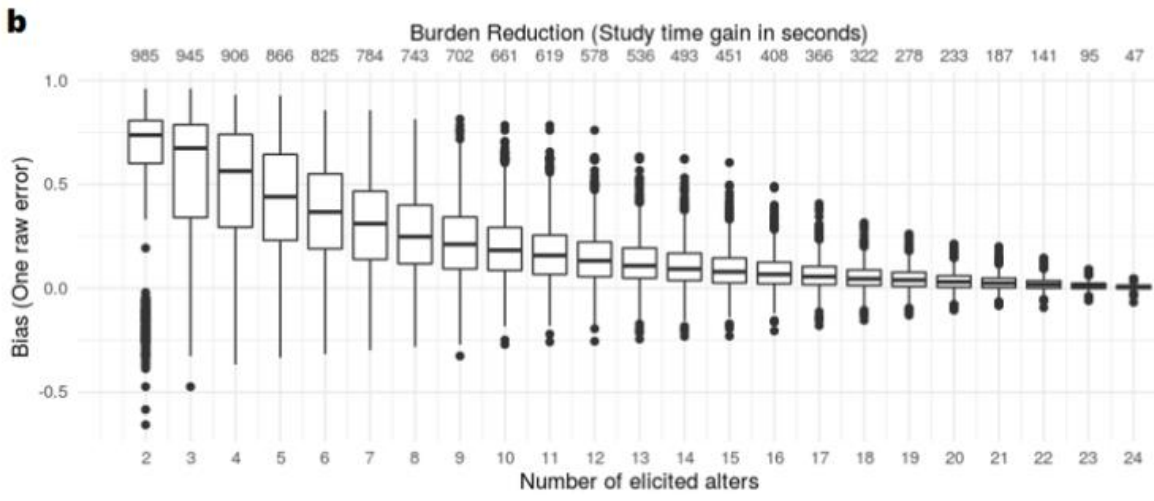

d

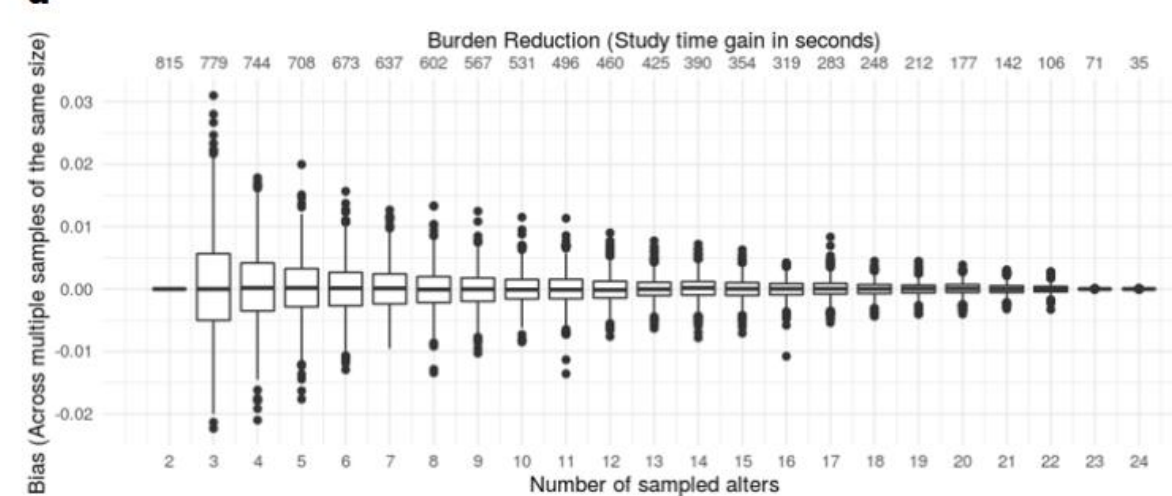

Note. $N=701$; Burden reductions are displayed for the average respondent of our reduced sample $(N=649)$. 


\section{Discussion}

In the present study we used two strategies to evaluate reductions in respondent burden in personal network studies connected to the number of alters that are included in the network: (1) eliciting and assessing fewer alters altogether and (2) eliciting a larger number of alters (i.e., 25) but only selecting a random subsample for answering name interpreter questions. We presented the amount of bias in structural and compositional network characteristics when applying these strategies as well as the respondent burden reduction (i.e., study time savings) for every possible network size (2 to 24 alters).

Our results show that there are considerable differences in how well different network characteristics can be estimated with the two methods for respondent burden reduction. Most measures that we investigated seem to be estimated better when eliciting 25 alters and then randomly sampling a subsample of those for full assessment rather than when dropping the last alters completely. This makes sense, since it can be assumed that respondents start with generating alter names of alters closer to them and with whom they have more contact. Thus, dropping the last alters, rather than selecting a random subsample of all generated names should bias the network towards more close social contacts. This assumption is confirmed by the sharp distinction between the two methods when considering some of the compositional characteristics of the networks (e.g., the proportion of kin and average closeness). According to our results, when a researcher is interested in estimating compositional network measures, eliciting 25 alters and then randomly sampling as few as 10 of those to assess alter attribute questions can be sufficient. This is especially useful since alter attribute questions take up most of the data collection time.

A similar, but less pronounced difference between the methods can be seen for structural characteristics such as network density, although structural measures generally seem to be more difficult to estimate than compositional ones. McCarty and colleagues (2007) conclude that when initially eliciting 45 alters, randomly selecting 10 alters is sufficient to estimate many structural characteristics. Based on our results, when eliciting only 25 alters initially, sampling fewer than 15 alters is not recommended when structural network measures are the main focus of study.

Particularly maximum degree and degree centralisation seem to be challenging to reliably estimate, corroborating earlier research by Golinelli and colleagues (2010). Results of McCarty and colleagues (2007) show similar issues, but only when estimating maximum degree based on a sample of fewer than 30 of their 45 elicited alters. This indicates that estimating this network measure without substantial bias requires researchers to elicit larger numbers of alters than 25 . The same seems to apply to degree centralisation, as it, contrary to our and Golinelli and colleagues' results, appeared quite stable in McCarty and colleagues' analyses when they estimated it based on any random alter sample drawn from 45 elicited alters.

Based on the distinction between compositional and structural measures, a possible respondent burden reduction strategy could be to, for example, elicit 25 alter names, then assess alter-to-alter ties 
for a random sample of 20 alters and then further randomly sample 10 alters of those 20 to assess alter attribute questions. Applying this strategy to the current network data collection would lead on average to a study duration of $11 \frac{1}{1} 2$ instead of 19 minutes.

A somewhat surprising result of our study is that in order to estimate the proportion of isolates, betweenness centralisation and maximum betweenness centrality eliciting fewer alters (i.e., 15 to 20) seems to perform better than randomly sampling from a previously generated alter list containing 25 alters. The results of McCarty and colleagues (2007) do only partially corroborate these findings: While maximum betweenness was also better estimated when simply dropping alters, their findings indicate that the proportion of isolates as well as betweenness centralisation is estimated better when randomly sampling alters rather than dropping alters completely. This difference may, again, be due to their larger initially elicited alter list.

The results of this this paper can aid researchers to strike a balance between burdening the respondent and obtaining reliable estimates for their particular compositional and structural network characteristics of interest (e.g., the proportion of kin in the network and network density). All results can be interactively explored in our accompanying shiny app (https://socialsciencemethods.shinyapps.io/BalancingBiasAndBurden/).

\section{Limitations and Future Research}

There are several aspects to keep in mind when using the results of this study. First, the present study is based on the assumption that researchers want their collected personal network to be representative of the whole social environment of the ego. This may not always be the case and it is important to match the data collection strategy to the underlying research questions: If only networks of confidants or frequent interaction partners are of interest, a small number of alters may be sufficient. If a network representative of the wider social environment (also including weak ties) is desired, the current results can be of help. However, some authors (e.g. Hogan et al., 2007) argue that not even 60 alters can provide such estimations since true personal networks contain multiple hundreds of social contacts.

Second, when comparing our results with the study by McCarty and colleagues (2007) it becomes apparent that the number of initially elicited alters matters. It would be useful for future research to obtain a network dataset with a larger list of initially elicited alters (e.g., 45 instead of 25) and then also investigate how long the list of generated alter names from which the random alter samples are drawn ideally should be. This could be done by gradually decreasing the generated alter list by dropping the last alter and at each step sampling random subsamples of all sizes in which network characteristics are determined. For example, first consider all 45 generated alters and then determine network measures in random samples of 2 to 44 of those 45 alters, then consider only the first 44 generated alters and determine network measures in random samples of 2 to 43 of those alters and so on. 
Third, while our sample is representative for Dutch women of reproductive age, it is unclear how far our results generalise beyond that. Particularly personal networks of older individuals differ from those of younger ones (Suanet et al., 2013). Older individuals might similarly take longer coming up with names and filling out the survey. Thus, when using our results, researchers should consider how well their target population matches our sample.

Lastly, structural and compositional network characteristic are seldom the final product of personal network research. They are often used as predictors in regression or multilevel models. From these results it is unclear how much error would be incurred in regression coefficients. Future studies should investigate the impact of respondent burden reduction strategies on conclusions regarding precise personal network research questions by performing full analyses with complete data as well as smaller alter samples.

\section{Conclusion}

The present study provides information on how to reduce respondent burden while minimising bias in the network characteristics a researcher would like to determine. In most cases eliciting 25 alters and then randomly sampling 15 to 20 of those for a full network assessment is a feasible strategy providing sufficiently precise estimates of network characteristics. However, there are considerable differences in how well different network measures can be estimated with this respondent burden reduction method: For some compositional measures even fewer alters may be sufficient, but for other structural measures simply eliciting fewer alters (i.e., 15 to 20) may perform just as well or even better.

Thus, researchers with an applicable target population should carefully consider the measures they are interested in and consult our results when deciding on the number of alters to elicit and assess. Our online tool (https://socialsciencemethods.shinyapps.io/BalancingBiasAndBurden) can aid this process. A particularly useful strategy to minimise respondent burden while minimising bias in estimates for researchers interested in both structural and compositional network characteristics could be to elicit a large number of alters, assess alter-to-alter ties for a random sample of those alters and then assess alter attributes for an even smaller subsample. In addition to limiting the number of alters, researchers should also consider to keep the number of particularly demanding attribute questions to a minimum in order to reduce study time and therefore respondent burden. 


\section{References}

Aschbrenner, K. A., Bobak, C., Schneider, E. J., Naslund, J. A., Brunette, M. F., \& O’Malley, A. J. (2018). Egocentric social networks and smoking among adults with serious mental illness. Translational Behavioral Medicine, 8(4), 531-539. https://doi.org/10.1093/tbm/ibx014

Bates, D., Maechler, M., Bolker, B., Walker, S., Christensen, R. H. B., Singmann, H., Dai, B., Grothendieck, G., Green, P., \& Bolker, M. B. (2015). Package 'lme4'. Convergence, 12(1), 2.

Buijs, V. L., \& Stulp, G. (2019). Friends, Family, and Family Friends: Predicting Friendships of Dutch Women [Preprint]. Open Science Framework. https://doi.org/10.31219/osf.io/y5dh7

Coromina, L., \& Coenders, G. (2006). Reliability and validity of egocentered network data collected via web: A meta-analysis of multilevel multitrait multimethod studies. Social Networks, 28(3), 209-231. https://doi.org/10.1016/j.socnet.2005.07.006

Costenbader, E., \& Valente, T. W. (2003). The stability of centrality measures when networks are sampled. Social Networks, 25(4), 283-307. https://doi.org/10.1016/S0378-8733(03)00012-1

Csardi, M. G. (2013). Package 'igraph’. Last Accessed, 3(09), 2013.

Eddens, K. S., Fagan, J. M., \& Collins, T. (2017). An interactive, mobile-based tool for personal social network data collection and visualization among a geographically isolated and socioeconomically disadvantaged population: early-stage feasibility study with qualitative user feedback. Jmir Research Protocols, 6(6), 124. https://doi-org.proxyub.rug.nl/10.2196/resprot.6927

Gerich, J., \& Lehner, R. (2006). Collection of Ego-Centered Network Data with Computer-Assisted Interviews. Methodology, 2(1), 7-15. https://doi.org/10.1027/1614-2241.2.1.7

Golinelli, D., Ryan, G., Green, H. D., Kennedy, D. P., Tucker, J. S., \& Wenzel, S. L. (2010). Sampling to Reduce Respondent Burden in Personal Network Studies and Its Effect on Estimates of Structural Measures. Field Methods, 22(3), 217-230. https://doi.org/10.1177/1525822X10370796

Granovetter, M. S. (1973). The Strength of Weak Ties. American Journal of Sociology, 78(6), 13601380.

Harrell, Jr, F. E. (2019). Package 'Hmisc'. CRAN2018, 235-6. 
Hogan, B., Carrasco, J. A., \& Wellman, B. (2007). Visualizing Personal Networks: Working with Participant-aided Sociograms. Field Methods, 19(2), 116-144. https://doi.org/10.1177/1525822X06298589

Hsieh, Y. P. (2015). Check the phone book: Testing information and communication technology (ICT) recall aids for personal network surveys. Social Networks, 41, 101-112. https://doi.org/10.1016/j.socnet.2014.11.006

Kinderman, P., Kamens, S., Robbins, B. D., \& Farley, B. D. (2020). Open letter regarding the reform and revision of diagnostic systems. https://Www.Apadivisions.Org. https://www.apadivisions.org/division-32/leadership/task-forces/diagnostic-alternatives

Lackaff, D. (2012). New Opportunities in Personal Network Data Collection. In M. Zacarias \& J. V. de Oliveira (Eds.), Human-Computer Interaction: The Agency Perspective (pp. 389-407). Springer. https://doi.org/10.1007/978-3-642-25691-2_17

Manfreda, K. L., Vehovar, V., \& Hlebec, V. (2004). Collecting Ego-centred Network Data via the Web. Metodoloski zvezki, 1(2), 295.

Marin, A., \& Hampton, K. N. (2007). Simplifying the Personal Network Name Generator: Alternatives to Traditional Multiple and Single Name Generators. Field Methods, 19(2), 163193. https://doi.org/10.1177/1525822X06298588

Marsden, P. (2014). Survey methods for network data. In The SAGE handbook of social network analysis (pp. 370-388). SAGE Publications Ltd, https://www-doi-org.proxyub.rug.nl/10.4135/9781446294413

Matzat, U., \& Snijders, C. (2010). Does the online collection of ego-centered network data reduce data quality? An experimental comparison. Social Networks, 32(2), 105-111. https://doi.org/10.1016/j.socnet.2009.08.002

McCarty, C., \& Govindaramanujam, S. (2005). A modified elicitation of personal networks using dynamic visualization. Connections, 26(2), 61-69.

McCarty, C., Killworth, P. D., \& Rennell, J. (2007). Impact of methods for reducing respondent burden on personal network structural measures. Social Networks, 29(2), 300-315. https://doi.org/10.1016/j.socnet.2006.12.005 
McCarty, C., Lubbers, M. J., Vacca, R., \& Molina, J. L. (2019). Conducting Personal Network Research: A Practical Guide. Guilford Publications. Guilford Publications.

McCarty, C., Molina, J. L., Aguilar, C., \& Rota, L. (2007). A Comparison of Social Network Mapping and Personal Network Visualization. Field Methods, 19(2), 145-162. https://doi.org/10.1177/1525822X06298592

Pedersen, T. L. (2018). tidygraph: A Tidy API for Graph Manipulation. R Package Version, 1(0). Pedersen, Thomas Lin. (2017). ggraph: An implementation of grammar of graphics for graphs and networks. R Package Version 0.1, 1.

Pedersen, Thomas Lin. (2019). patchwork: The Composer of Plots (1.0.0) [Computer software]. https://CRAN.R-project.org/package=patchwork

Peregrine Documentation. (2020). https://wiki.hpc.rug.nl/peregrine/start

Perry, B. L., Pescosolido, B. A., \& Borgatti, S. P. (2018). Egocentric network analysis: Foundations, methods, and models (Vol. 44). Cambridge University Press.

R Core Team, R. (2018). R: A language and environment for statistical computing. R Foundation for Statistical Computing. Austria: Vienna.

Rapp, C., Ingold, K., \& Freitag, M. (2019). Personalized networks? How the Big Five personality traits influence the structure of egocentric networks. Social Science Research, 77, 148-160. https://doi.org/10.1016/j.ssresearch.2018.09.001

Robinson, D. (2014). broom: An R package for converting statistical analysis objects into tidy data frames. ArXiv Preprint ArXiv:1412.3565.

Ryan, G. W., Stern, S. A., Hilton, L., Tucker, J. S., Kennedy, D. P., Golinelli, D., \& Wenzel, S. L. (2009). When, Where, Why and with Whom Homeless Women Engage in Risky Sexual Behaviors: A Framework for Understanding Complex and Varied Decision-Making Processes. Sex Roles, 61(7-8), 536-553. https://doi.org/10.1007/s11199-009-9610-z

Scherpenzeel, A. (2011). Data Collection in a Probability-Based Internet Panel: How the LISS Panel Was Built and How It Can Be Used. Bulletin of Sociological Methodology/Bulletin de Méthodologie Sociologique, 109(1), 56-61. https://doi.org/10.1177/0759106310387713 
Stadel, M.; Stulp, G, 2020, "Analysis Scripts for 'Balancing Bias and Burden in Personal Network Studies'", https://doi.org/10.34894/JNVWNH, DataverseNL, DRAFT VERSION.

Stark, T. H., \& Krosnick, J. A. (2017). GENSI: A new graphical tool to collect ego-centered network data. Social Networks, 48, 36-45. https://doi.org/10.1016/j.socnet.2016.07.007

Stulp, G. (2020). Collecting large personal networks in a representative sample of Dutch women. Social Networks, 64, 63-71.

Suanet, B., Van Tilburg, T. G., \& Broese van Groenou, M. I. (2013). Nonkin in older adults' personal networks: more important among later cohorts?. Journals of Gerontology Series B: Psychological Sciences and Social Sciences, 68(4), 633-643.

Tubaro, P., Casilli, A. A., \& Mounier, L. (2014). Eliciting Personal Network Data in Web Surveys through Participant-generated Sociograms. Field Methods, 26(2), 107-125. https://doi.org/10.1177/1525822X13491861

Vehovar, V., Lozar Manfreda, K., Koren, G., \& Hlebec, V. (2008). Measuring ego-centered social networks on the web: Questionnaire design issues. Social Networks, 30(3), 213-222. https://doi.org/10.1016/j.socnet.2008.03.002

Waring, E., Quinn, M., McNamara, A., Rubia, E. A. de la, Zhu, H., Lowndes, J., Ellis, S., McLeod, H., Wickham, H., Müller, K., RStudio, functions), I. (Spark, Kirkpatrick, C., Brenstuhl, S., Schratz, P., lbusett, Korpela, M., Thompson, J., McGehee, H., ... Possenriede, D. (2020). skimr: Compact and Flexible Summaries of Data (2.1.1) [Computer software]. https://CRAN.R-project.org/package=skimr

Wasserman, S., \& Faust, K. (1994). Social network analysis: Methods and applications (Vol. 8). Cambridge university press.

Wickham, H. (2016). ggplot2: Elegant graphics for data analysis. Springer.

Wickham, H. (2017). Package tidyverse. Easily Install and Load the 'Tidyverse.

Xie, Y. (2014). knitr: A comprehensive tool for reproducible research in R. Implementing Reproducible Computational Research, 3-32. 
${ }^{\mathrm{i}}$ Closeness and betweenness centralisation, as well as betweenness centrality are not defined for less than 3 alters, thus for those measures emulated network sizes only range from 3 to 24 alters.

ii Emulating 10.000 samples did not change results significantly. 